\title{
SCHIZOPHRENIA LIKE PSYCHOSIS IN PATIENTS WITH EPILEPSY CASE REPORT
}

Ranjan Sanjeev ${ }^{1}$, Chalise Pratikshya ${ }^{2}$, Pandey Pradeep², Poudel Reet', Aich T.K. ${ }^{3}$

\begin{abstract}
Epilepsy and schizophrenia are both due to altered cerebral functioning and their history is often connected. Here we present a case of epilepsy of generalized tonic clonic type presenting with schizophrenia like symptoms.
\end{abstract}

KEYWORDS: Epilepsy, schizophrenia like psychosis.

\section{INTRODUCTION}

Schizophrenia and epilepsy sometimes concur in the same person. In the 1950s, investigators found that patients with epilepsy, particularly those with temporal lobe epilepsy, suffered from a psychotic disorder similar to schizophrenia. However, the symptoms of psychosis in patients with epilepsy differed in many ways from schizophrenia. Slater et al. coined the term "schizophrenia-like psychosis" for such psychosis. It was noted that although such patients shared other clinical features typical of schizophrenia, they did not display the lack of affect and withdrawn attitude that were typical of the schizophrena. ${ }^{1}$

In the present classifications for the diagnosis of mental disorders, these patients would receive two different diagnoses without contemplating the possibility that they are related disorders.

Although schizophrenia like psychosis is frequently reported with temporal lobe epilepsy, its occurrence is not so frequently reported in epilepsy of generalized tonic clonic type.

1. Associate Professor, Department of Psychiatry, Universal College of Medical Sciences \& Teaching Hospital, Bhairahawa, Nepal.

2. Post Graduate Resident, Department of Psychiatry, Universal College of Medical Sciences \& Teaching Hospital, Bhairahawa, Nepal.

3. Professor, Department of Psychiatry, Universal College of Medical Sciences \& Teaching Hospital, Bhairahawa, Nepal.

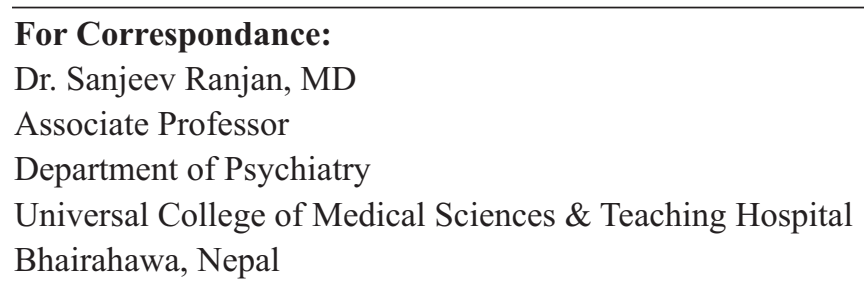




\section{CASE REPORT}

A 32 year old married male from Dang, labor by occupation, presented to the psychiatry outpatient department of universal college of medical science with chief complain of fearfulness, hearing and seeing things which others could not see and irritability for two months. History of present illness was suggestive of third person auditory hallucination, visual hallucination, bizarre delusion, wandering away from home to jungle, somatic hallucination, delusion of control, delusion of persecution, delusion of reference, insomnia and neglect of personal hygiene. He had past history of loss of consciousness six years back during sleep, lasting for 1-2 minutes, associated with rapid jerky movements of all four limbs, frothing from mouth, clenching of teeth, up rolling of eye balls, tongue bite and post ictal confusion. He was diagnosed as a case of epilepsy and was started on antiepileptic drugs, the name of which patient does not remember and no documents were available. He took that medicine for three years. Thereafter he stopped medicine on his own and was asymptomatic. Eight months back he had similar episode during sleep and was brought to the emergency of this medical college. He was started on phenytoin $300 \mathrm{mg}$ at night. He was under regular medication since then. He had family history of epilepsy in his nephew who is also under medication since three years. He had well-adjusted premorbid personality and no history of substance abuse.

On examination, patient was conscious, well oriented with no abnormality detected on general physical examination and on systemic examination. Mental status examination revealed presence of retardation of thinking, bizarre delusion, third person auditory hallucination, somatic hallucination and ill sustained concentration with partial insight. His baseline investigations were normal. He was diagnosed as a case of epilepsy with schizophrenia like psychosis. Brief Psychiatry Rating Scale (BPRS) was applied initially at the time of admission and the score was 47. A computed tomography (CT) scan and MRI of the brain was done and was found to be normal.

Patient was initially on Phenytoin at the time of admission and to rule out phenytoin induced psychosis he was switched to sodium valproate $(1000 \mathrm{mg})$ and though seizure was under control the psychotic symptoms did not subside with it. Then clobazam $(10 \mathrm{mg})$ was added to see whether those symptoms were occurring in ictal phase of seizure, the symptoms again persisted. So phenytoin induced psychosis was ruled out. Patient was then put on olanzapine $(20 \mathrm{mg})$ after which patient gradually returned to the baseline state. BPRS was again applied and it was found to be 17 .

\section{DISCUSSION}

The relationship between schizophrenia and epilepsy has been of great interest. Prevalence of schizophrenia in the interictal period of epilepsy varies between studies; from 6-12\%. ${ }^{2}$ Most study shows high prevalence in temporal lobe epilepsy. Greater susceptibility to both illnesses could be due to similar or shared genetic, environmental or neurobiological causes. Psychotic disorders in patients with epilepsy are often overlooked, mistreated, and consequently lingering on needlessly. While early diagnosis is unanimously supported as a first step to avoid this delay, necessity of switching from antiepileptic drugs with supposedly adverse psychotropic effects to others is more controversial. Most cases of schizophrenia were reported in patients with temporal lobe epilepsy, and lesions in the temporal lobe were found in many of the cases. ${ }^{3}$ In our case, patient had generalized tonic clonic seizure and no lesion was found in temporal lobe in brain imaging.

Average interval between the onsets of epilepsy n psychosis in most studies range from 10-15 years. ${ }^{2}$ In our study, the interval was of six month.

\section{CONCLUSION}

Schizophrenia is associated not only with temporal lobe epilepsy but also with generalized tonic clonic seizure without any lesion in the temporal lobe.

\section{REFERENCES}

1. Kanner MA. "Psychosis of Epilepsy”. Epilepsy \& Behavior 1, 219-227 (2000) doi:10.1006/ebeh.2000.0090.

2. Bersani G, Iannitelli A, Quartini A, Di Blasi C, Gualdi G, Pancheri P. "Patients with epilepsy associated with schizophrenia: a descriptive study of patients investigated with MRI and standard EEG”. 2008; 14:10-15.

3. Yalug I, Tufan AE, Kutlu H, Alemdar M. "Temporal lobe epilepsy and psychosis: a case report”. New/Yeni Symposium Journal. 2007; 17:46. P 41 . 